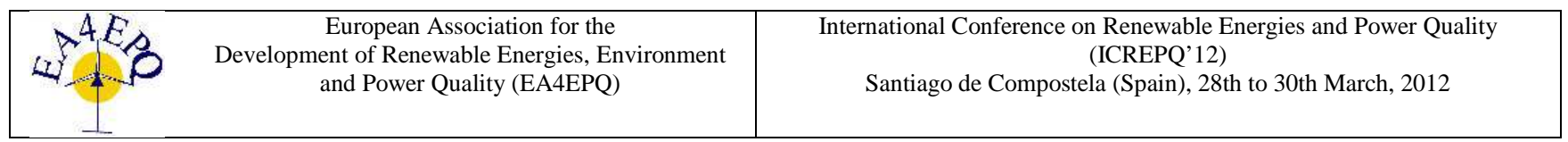

\title{
Comparison of Multi-Resonant- and Hysteresis Band Controllers used in Current Control Loop of Shunt Active Power Filter
}

\author{
R. Pavlanin ${ }^{1}$, P. Spanik ${ }^{1}$ and B. Dobrucky ${ }^{1}$ \\ ${ }^{1}$ Department of Mechatronics and Elektronics \\ University of Zilina \\ Univerzitna 8215/1, 01026 Zilina (Slovakia) \\ Phone/ number: +421 41513 1601, Fax: +421 415131524 \\ E-mail: 1pavlanin@fel.uniza.sk, dobrucky@fel.uniza.sk, spanik@fel.uniza.sk
}

\begin{abstract}
In the paper the advantages and disadvantages of both current control loops with hysteresis and resonant types are presented by simulation analyses and experimental results. The result of comparison defines some recommendations for the fields of use of shunt active power filter controlled by these regulators.
\end{abstract}

\section{Key words}

Shunt active power filter, hysteresis controller, resonant type controller

\section{Introduction}

Shunt active power filters (SAPF) have been developed to eliminate of harmonics and reactive current components caused by non-linear load connected to the network. Regardless to control method [1]-[6], for reference current determination they comprise two control loops: voltage and current ones. To ensure well filter current control the voltage on DC bus has to be bigger than the maximum of instantaneous voltage of network during whole period of fundamental component. Thus DC bus voltage control loop is necessary. The DC bus voltage is controlled by controlling the magnitude of fundamental current component of filter (small amount of active power of SAPF). According to this fact the voltage control loop can be much slower than current control loop to avoid filter current disturbances. Hence both control loops can be designed separately.

\section{SAPF current control loop}

One of the most important of the control parts of shunt active power filter is a current control loop. It is used regardless to that if direct or indirect methods is used [2], [3], [5] - one of them is in Fig. 1.
The simplified block diagram of current loop of SAPF from the point of view of the feedback control loop is shown in Fig. 2.

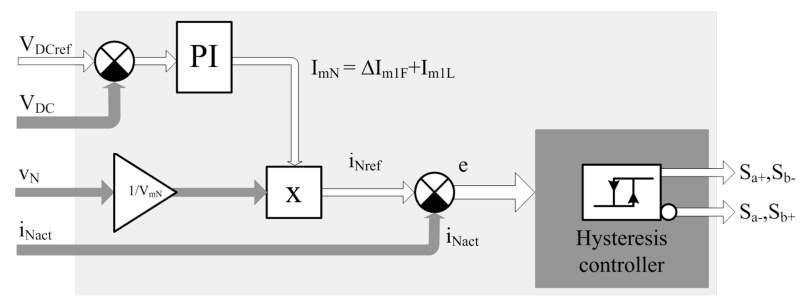

Fig. 1 Block diagram of direct network current control

Considering the voltage source inverter in power circuit of SAPF the output is a controllable voltage $V_{\mathrm{F}}$. Because of current control of SAPF it is necessary to connect coupling inductance between filter and network.

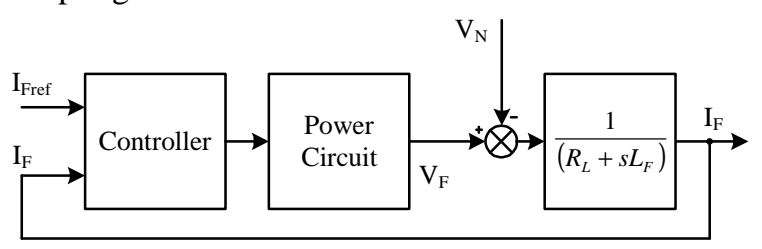

Fig. 2 Block diagram of current control loop of SAPF

According to equation of the system in Laplace form:

$$
V_{F}(s)=V_{N}(s)+\left(R_{L}+s L_{F}\right) I_{F}(s)
$$

The filter current $I_{\mathrm{F}}(\mathrm{s})$ can be derived from equation (1):

$$
I_{F}(s)=\frac{V_{F}(s)-V_{N}(s)}{\left(R_{L}+s L_{F}\right)}
$$

where $V_{\mathrm{F}}$ is inverter output voltage, $V_{\mathrm{N}}$ is the voltage at PCC, $L_{\mathrm{F}}$ is a coupling inductance and $R_{\mathrm{L}}$ is its resistance. In the following, the hysteresis band controller and resonant controller structures for the shunt active power filter are discussed. The implementation of the control strategies for such as this systems can be done in different reference frames (phase reference frame $a b c$, 
stationary $\alpha-\beta$ or synchronous rotating $d-q$ ). Because of one phase system we focus our interest to study a difference between simple hysteresis band controller and resonant controller in one phase reference frame.

\section{Current control loop with hysteresis band controller}

The hysteresis band controller represents the simplest way of current control of shunt active power filter with very good dynamics response [4]-[6]. The basic principle of hysteresis band controller is shown in Fig. 3 .

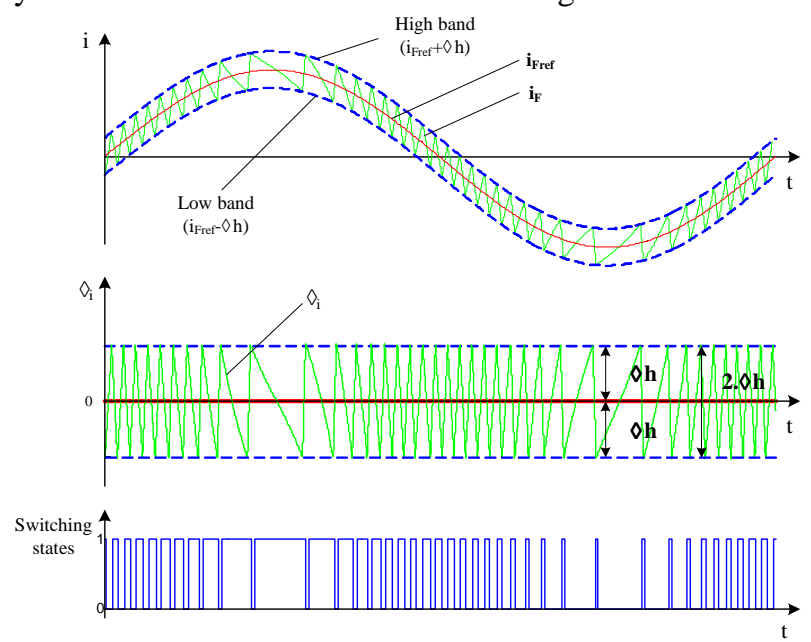

Fig. 3 Principle of hysteresis controller

The error between reference current and actual current is maintained in a certain current band commonly known as hysteresis band $(2 \Delta h)$. Constant width of hysteresis band $\Delta h$ leads to asynchronous switching of power elements of SAPF i.e. variable switching frequency (see also Fig. 3 below). Variable switching frequency is disadvantage of hysteresis band current control. It causes a problem of EMI filter design of SAPF, possibility of unacceptable resonances generation and so on.

A block diagram of current control loop with hysteresis band controller is shown in Fig. 4.

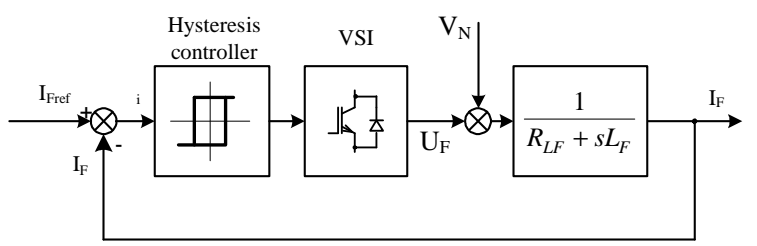

Fig. 4 Block diagram of current control loop with hysteresis controller

At first measured actual value of filter current $i_{\mathrm{F}}(\mathrm{t})$ is compared with reference current value $i_{\text {Fref }}(\mathrm{t})$. Switching process does not change until error signal $\square_{i}$ is inside of hysteresis band (between higher and lower band). Appropriate switching process is produced when error signal exceeds higher or lower hysteresis band of controller. It is clear that the computing time of hysteresis band controller implemented in digital control unit will be quite sort but on the other hand sampling frequency of measured current has to be as big as possible (or much more bigger than switching frequency). This is the second main disadvantage of hysteresis band controller. There are a number of techniques to limit the maximum switching or sampling frequency of hysteresis band controller. Limit of these frequencies definitely has to affect uniformity of hysteresis band ( $\Delta h \neq$ constant).

\section{Current control loop with a multi- resonant controller}

The main disadvantages of hysteresis band current controller can be eliminated using carrier based PWM techniques. A modulating voltage reference wave has been supplied from multi-resonant controller (Fig. 5).

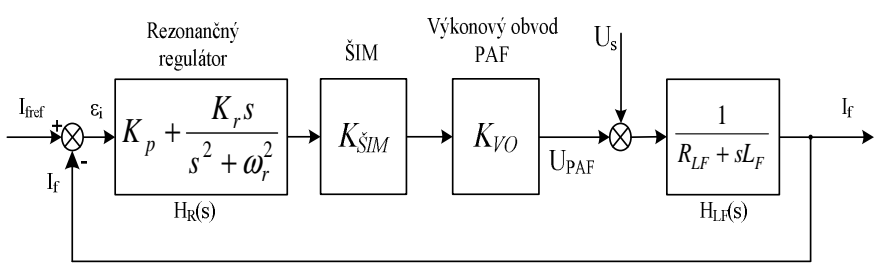

Fig. 5 Block scheme of current loop with resonant controller

The principle of multi-resonant controller is based on ideal resonant circuit. The transfer function of ideal (lossless) resonant circuit can by defined by:

$$
H_{R}(s)=\frac{s}{s^{2}+\omega_{r}^{2}}
$$

where $\omega_{\mathrm{r}}$ represents resonant frequency. Then the transfer function of resonant controller [14] is defined by:

$$
H_{R C}(s)=K_{p}+K_{r} \frac{s}{s^{2}+\omega_{r}^{2}}
$$

where $K_{\mathrm{p}}$ represents proportional gain and $K_{\mathrm{r}}$ represents gain of resonant element.

Bode diagram of resonant controller with different gains of resonant element is shown in Fig. 6. As it can be seen from the figure and also Eq. (2) all differences between reference and actual current with a resonant frequency wave are amplified with theoretically infinite gain. Proportional gain $K_{\mathrm{p}}$ of resonant controller is added to reduce the effect of resonant gain $K_{\mathrm{r}}$. Block diagram of current control loop of SAPF with resonant controller shown in Fig. 5 is just for one harmonic current component control. Where $K_{\mathrm{PWM}}$ represents gain of PWM and $K_{\mathrm{PC}}$ represents DC bus gain.

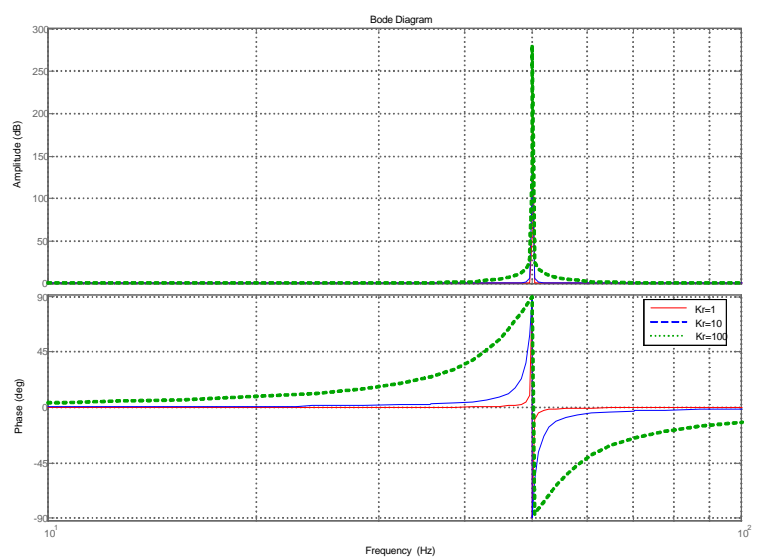

Fig. 6 Bode diagram for different gains $K_{\mathrm{r}}\left(\omega_{\mathrm{r}}=2 \pi 50 \mathrm{r.s} \mathrm{s}^{-1}\right)$

For the open loop transfer function is valid:

$$
H_{0}(s)=K_{\check{S} I M} K_{V O} \frac{K_{p} s^{2}+K_{r} s+K_{p} \omega_{r}^{2}}{L_{F} s^{3}+R_{L F} s^{2}+L_{F} \omega_{r}^{2} s+R_{L F} \omega_{r}^{2}}
$$


If considering unity of $\mathrm{K}_{\mathrm{PWM}}$ and $\mathrm{K}_{\mathrm{PC}}$ then the close loop transfer function yields:

$$
H(s)=\frac{\frac{K_{p}}{L_{F}} s^{2}+\frac{K_{r}}{L_{F}} s+\frac{K_{p}}{L_{F}} \omega_{r}^{2}}{s^{3}+\frac{\left(K_{p}+R_{L F}\right)}{L_{F}} s^{2}+\frac{\left(K_{r}+\omega_{r}^{2} L_{F}\right)}{L_{F}} s+\frac{\left(K_{p}+R_{L F}\right)}{L_{F}} \omega_{r}^{2}}
$$

Pole placement method can be used for setting of optimal parameters of controller. Location of poles and zeros and also bode diagram for different proportional components $K_{\mathrm{p}}$ of controller are shown in Fig. 7a. Effect of resonant gains $\mathrm{K}_{\mathrm{r}}$ setting is shown in Fig. 7b. According to harmonic components present in reference current of SAPF, more resonant components have to be used in current controller. Each resonant component of controller should be tuned to harmonic component to be compensated by SAPF. The transfer function of such this controller commonly known as multi-resonant controller is given by:

$$
H_{M R}(s)=K_{p}+\sum_{h=1,3,5,7 \ldots}^{n} \frac{K_{r h} s}{s^{2}+\left(h \cdot \omega_{r}\right)^{2}}
$$

where $h$ represents number of harmonic component.

For example of one phase SAPF: for compensation of reactive power and for DC bus voltage control the controller has to have resonant component tuned on fundamental component $(h=1)$, for distortion power compensation controller has to have resonant component tuned to $3^{\text {rd }}, 5^{\text {th }}, 7^{\text {th }}$ etc. harmonic components $(h=3,5,7$, ...), it is depending on application. It has to be noted that number of resonant components used in multi-resonant controller markedly influence computing power of control unit.

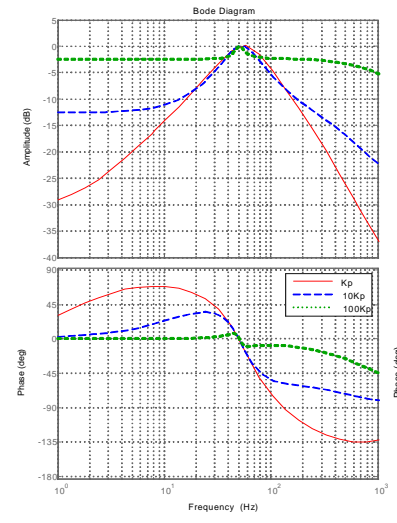

a

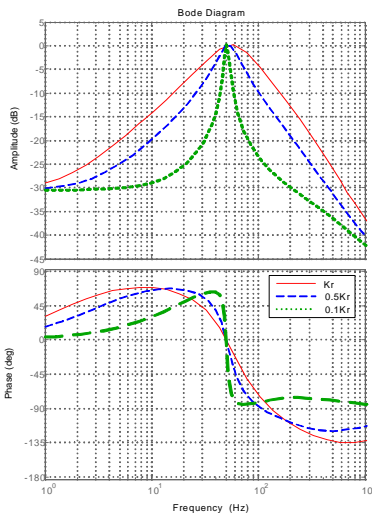

b
Fig .7 Bode diagrams a. effect of proportional gain $\mathrm{K}_{\mathrm{p}}$, b. effect of resonant gain $\mathrm{K}_{\mathrm{r}}$

\section{Simulation model in Matlab / Simuling}

Parameters of simulation model including power circuit (network, SAPF and load) and control part are:

Parameters of network: magnitude of voltage $100 \mathrm{~V}$; frequency $50 \mathrm{~Hz}$; THD voltage $3.3 \%$.

Parameters of rectifier load (continuous mode): filter inductance $44 \mathrm{mH}$; load resistance $24.3 \Omega$.

Parameters of rectifier load (discontinuous mode): filter capacitance $800 \mu \mathrm{F}$; filter inductance $12 \mathrm{mH}$; load resistance $38.6 \Omega$.

Parameters of SAPF:
- Power circuit

coupling inductance $12 \mathrm{mH}$

DC bus capacitance $780 \mu \mathrm{F}$

reference DC bus voltage $150 \mathrm{~V}$

no EMI filter used

- Control part - Hysteresis controller

switching frequency (limited up to $12 \mathrm{kHz}$ ),

sampling frequency optimized up to $\sim 160 \mathrm{kHz}$.

- Control part - Multi-resonant controller

four resonant components (fundamental, $3^{\text {rd }}, 5^{\text {th }}$ and $7^{\text {th }}$ ) switching frequency $5 \mathrm{kHz}$ (modulating frequency of inverter voltage $10 \mathrm{kHz}$ )

sampling frequency $20 \mathrm{kHz}$.

Note: Ideal voltage source to simulate the network was used in simulation model.

Fig. 8 and Fig. 9 show result of simulation analyses. The figures show comparison between hysteresis band controller (Fig. 8a, Fig. 9a) and multi-resonant controller (Fig. 8b, Fig. 9b) used in current control loop of SAPF. Each of them presents steady state of compensation of one phase full bridge diode rectifier working in continuous (Fig. 8) and discontinuous mode (Fig. 9). Just fundamental, $3^{\text {rd }}, 5^{\text {th }}$ and $7^{\text {th }}$ resonant component has been used in a structure of multi-resonant controller to demonstrate its basic characteristics.
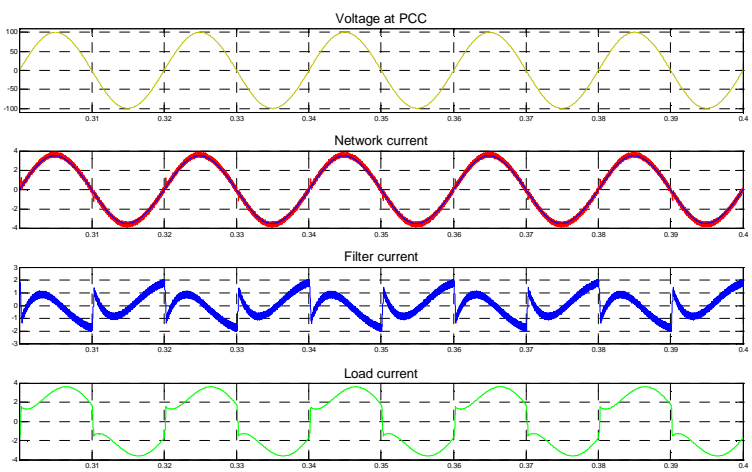

a) hysteresis controller
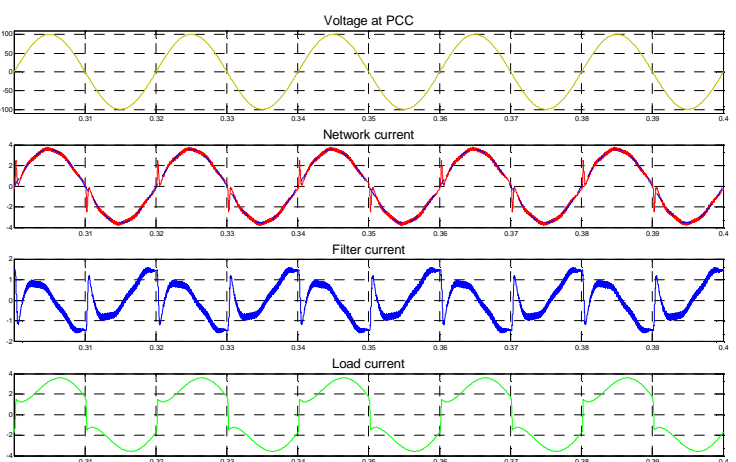

b) resonant controller

Fig. 8 Compensation of rectifier load (continuous current mode)

A current spike presents in the network current (most visible in Fig. 8b) are caused by rectifier diodes commutation process. Hysteresis band controller had not such a big problem with compensation of these current spikes due to its excellent dynamic properties. 

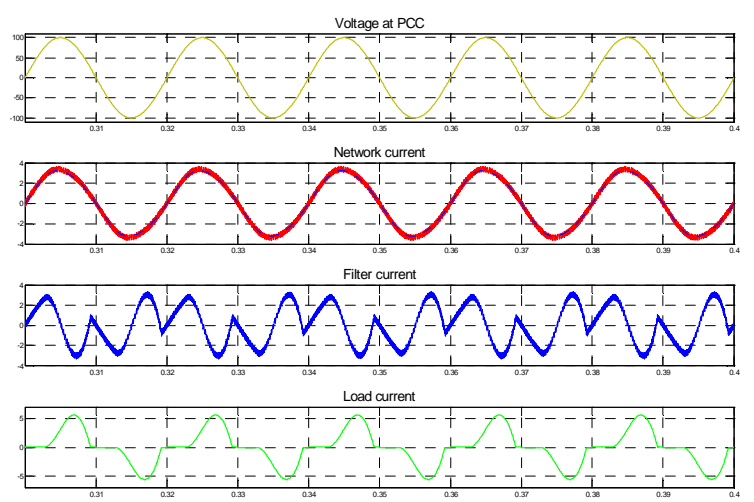

a) hysteresis controller

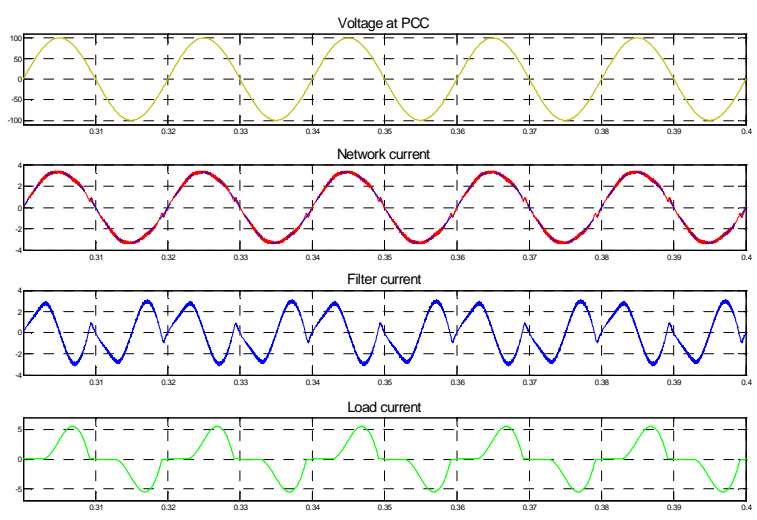

b) resonant controller

Fig. 9 Compensation of rectifier load (discontinuous mode)

As it can be seen in Fig. 8b and also in Fig.9b multiresonant controller had a problem with compensation of bigger current derivations (di/dt).

On the other hand multi-resonant controller fails to compensate these spikes. This fact was cause because multi-resonant controller was tuned to only four harmonic components $\left(1^{\text {st }}, 3^{\text {rd }}, 5^{\text {th }}\right.$ and $\left.7^{\text {th }}\right)$. So, the current spike caused by commutation process in diode rectifier was completely attenuated in resonant controller due to its high frequency. This is the one of the biggest disadvantages of multi-resonant controller used in current control loop of SAPF. Multi-resonant controller used mainly in such these applications of power compensators has multiple advantages compare to hysteresis band controller. The most important advantage is a constant switching frequency and also much smaller sampling frequency. Design of passive filter for switching frequency attenuation at PCC is much simpler.

\section{Experimental verification}

Parameters for experiments used are given above (in simulation analysis section).

One phase of 3-phase full-bridge VSI has been used as a power circuit. In a case of multi-resonant controller unipolar switching technique has been used (modulation frequency of output voltage is two time bigger than switching frequency of IGBTs). Powerful digital signal processor TMS320F2808 [5], [7], [8] was used as a control unit. According to digital control hysteresis band controller has had limited sampling (as great as possible) and also switching frequency.

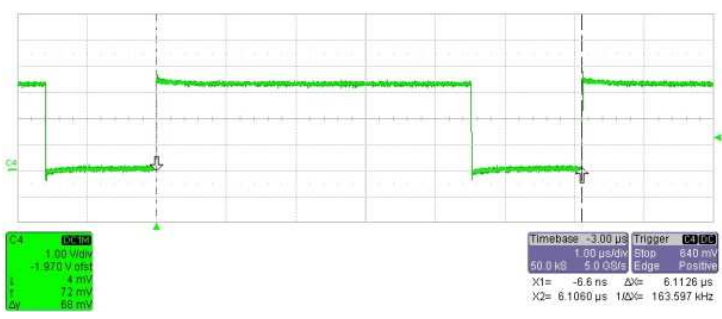

a) hysteresis band controller

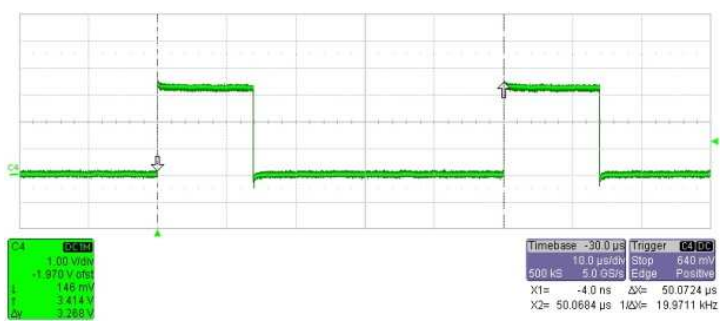

b) resonant controller

Fig. 10 Comparison of computing time and sampling frequency of two types of controllers

Fig. $11(a, b)$ shows a comparison of computation times and sampling frequencies between hysteresis band controller and multiresonant controller. Duration of high level in the picture represents the computing times and the period represents sampling frequency. As it can be seen the sampling frequency of hysteresis band controller $(\sim 163 \mathrm{kHz})$ is more than eight times greater than in a case of multi-resonant controller $(19.97 \mathrm{kHz})$. There was no place for reduction of sampling frequency in a case of hysteresis band controller according to unacceptable waveform of filter current. On the other hand there was place for reduction of sampling frequency down to $5 \mathrm{kHz}$ in a case of multi-resonant controller using sampling in the middle of the switching pulse. In our case multisampling technique has been used (four samples for one switching interval).

The computation time in a case of hysteresis band controller (something about 4,5 $\mu \mathrm{s}$ ) was less than $1 / 3$ compare to computation time in a case of multi-resonant controller (something about $14 \mu \mathrm{s}$ ). It was impossible to increase sampling frequency to improve dynamic of hysteresis band controller because of its computation time. The computation time in that case was short because of very easy implementation of hysteresis band controller in the digital control. Four resonant components have been used in a case of multi-resonant controller.

Transfer functions for all these components (in Laplace form (7)) have been implemented in the digital control making z-transformation. Thus implementation of multiresonant controller has had greater impact on computing power compare to hysteresis band controller. However there was no need to increase sampling frequency to improve dynamics of multi-resonant controller. More resonant components have to be implemented into the multi-resonant controller to improve its dynamics. It has to be noted that computation time in these cases includes voltage control loop calculation and reference current calculation as well. 
Duration of low level in Fig. 11 a, b represents rest of computation time. In the case of multi-resonant controller there is a lot of time rest (more than $35 \mu \mathrm{s}$ ) compare to hysteresis band controller (not more than $1.5 \mu \mathrm{s}$ ). Hence more resonant component can be added in the case of multi-resonant controller to improve its dynamics. Definitely it has to be considered some rest time for other calculations. For example in the industrial applications there can be necessary to implement I2T protection, $R M S$ values calculation and $F F T$ calculation etc.

Experimental results of SAPF working with hysteresis band controller and multi-resonant controller in current control loop are shown in Fig. 12a, b, Fig.13a, b as a steady state of operation.

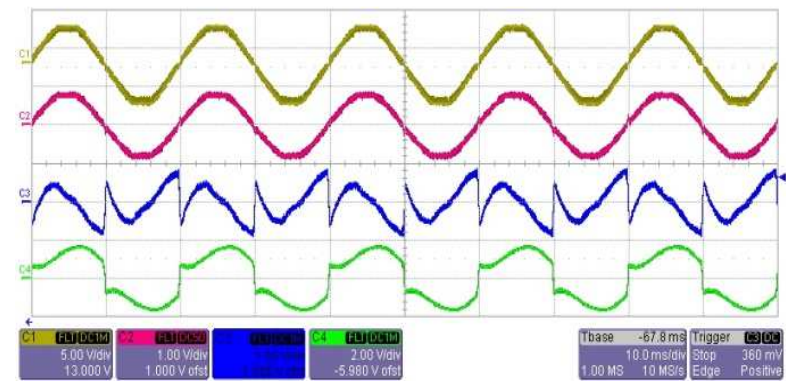

a) hysteresis band controller

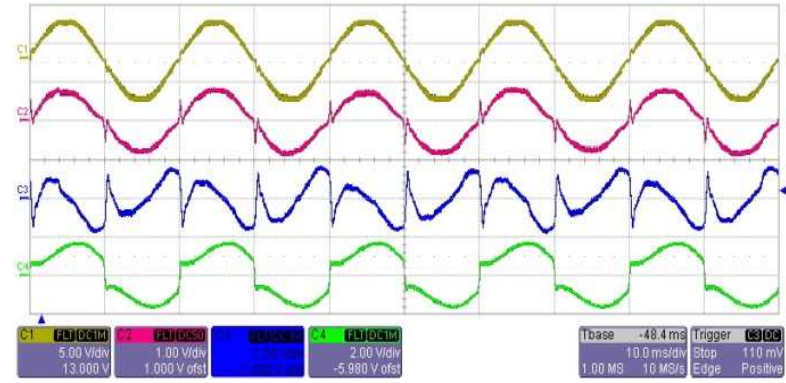

b) resonant controller

Fig. 12 Compensation of rectifier load (continuous current mode).

It can be seen the same results as in simulation analysis. Multi-resonant controller has a problem with current spikes produced by commutation process of rectifier diodes. The same comment can be used like in simulation analysis. There is no resonant component presents in multi-resonant controller structure can compensate these current spikes. There is no such a big difference between these two controllers in a case of compensation of rectifier load operating in discontinuous mode (Fig. 13).

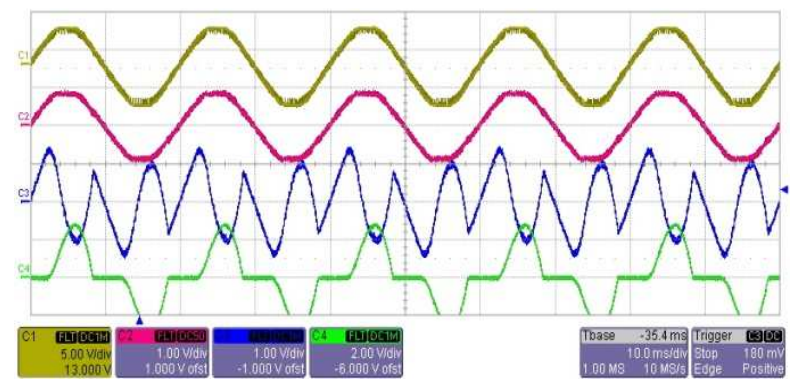

a. hysteresis band controller

Fig. 13a. Compensation of rectifier load (discontinuous current mode)

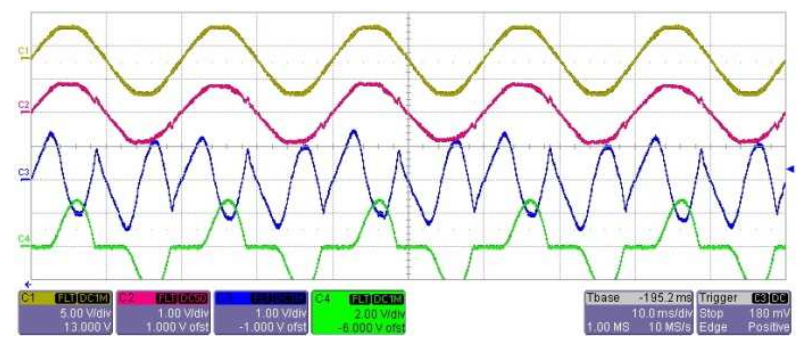

b. resonant controller

Fig. 13b. Compensation of rectifier load (discontinuous current mode)

\section{Conclusions}

In the paper comparison between hysteresis band controller and multi-resonant controller used in current control loop of SAPF is presented. Excellent dynamic properties (see in Fig. 8a, Fig. 9a, Fig. 12a, Fig.13a) and also simple implementation into the digital control are the biggest advantages of hysteresis band controller. Demand on control power of control unit may be misleading in a case of hysteresis band controller because of high sampling frequency (Fig. 11a). The main advantage of multi-resonant controller used in current control loop of SAPF is constant switching frequency compare to variable switching frequency produced by hysteresis band controller. This feature is preferred in industrial applications because of switching frequency passive filter design. There is no such a good dynamics in the case of multi-resonant controller with four resonant components as in hysteresis band controller. It can be seen in figures of simulation analysis (Fig. 8b and Fig. 9b) and in figures of experimental results (Fig. 12b, Fig. 13b). A better dynamics of multi-resonant controller can be achieved by adding more resonant component into the controller structure. Digital control with multi-resonant controller is more complex compared to hysteresis band controller but much lower sampling frequency in that case makes more place for others calculations (Fig.11).

\section{Acknowledgement}

R\&D operational program Centre of excellence of power electronics systems and materials for their components II. No. OPVaV-2009/2.1/02-SORO, ITMS 26220120046 funded by European regional development fund (ERDF) and VEGA project No. 1/0943/11.

\section{References}

[1] D Chen, S Xie: Review of the control strategies applied to active power filters. In: Proc. of IEEE Int'l Conf. on Electric Utility Deregulation, Restructuring and Power Technologies (DRPT'04), April 2004, Hong Kong (PRC), pp. 666-670.

[2] H J Azevedo, J M Ferreira, A P Martins, A S Carvalho: An Active Power Filter with Direct Current Control for Power Quality Conditioning. In: Proc. of EPCS'98 Conf. on Electric Power Components and Systems, 1532-5016, Vol. 36, No. 6, 2008, pp. $587-601$. 
[3] M I Milanes, E Romero, F Barrero: Comparison of Control Strategies for Shunt Active Power Filters in Three-Phase Four-Wire Systems. In: Renewable Energies and Power Quality, Feb 2006 (based on F Barrero, E Romero, M I Milanes: Control Strategies for Active Power Filters. Proc. of Int'l Conf. on ICREPQ'04, Barcelona (ES)), 8 pgs.

[4] S Meo, A Perfetto: Comparison of different control techniques for Active Power Filter Applications. In: Proc. of IEEE $4^{\text {th }}$ Int'l Caracas Conference on Devices, Circuits and Systems -ICCDCS, Apr 17-19, 2002, Aruba (Dutch Caribbean), pp. P016_1-P016_6.

[5] R Pavlanin, B Dobrucky, P Spanik: Investigation of Compensation Effect of Shunt Active Power Filter working under the non-sinusoidal voltage conditions. In: IREE Int'l Review of Electrical Engineering (IT), Vol. 3, Sep-Oct 2009, No. 5, pp. N/A, ISSN 1827-6660.
[6] S George, V Agarwal: A Novel DSP Based Algorithm for Optimizing the Harmonics and Reactive Power Under Non-Sinusoidal Supply Voltage Conditions. In: IEEE Trans. on Power Delivery, Vol. 20, No. 4, Oct 2005, pp. 2526-2533.

[7] B Mazari, F Mekri: Fuzzy hysteresis control and parameter optimization of a shunt active power filter. In: Journal of Information Science and Engineering, 2005, Vol. 21, No. 6, ISSN 1016-2364, pp. 1139-1156.

[8] P Chlebis, P Simonik, L Osmancik, P Moravcik, M Dostalik: Active Power Filter Controlled by Digital Signal Processor TMS 320F2812, In: Proc. of $16^{\text {th }}$ Int'l Conf. on Electrical Drives and Power Electronics, $\mathrm{H}$. Tatras (SK), Sep 24-26, 2007. 